\title{
Isolation, identification and characterization of endophytic bacteria antagonistic to Phytophthora palmivora causing black pod of cocoa in Malaysia
}

\begin{abstract}
Black pod rot is the most significant factor limiting production of cocoa (Theobroma cacao) in Malaysia with average annual losses of above 30\%. This work was carried out to isolate, characterize and screen bacterial endophytes from cocoa plants for their biological control activities. Their mechanisms of action as well as abilities to reduce black pod rot disease were also investigated. In total, 103 endophytic bacterial isolates were obtained from healthy cocoa tissues (leaves, branches and fruits) from seven states of Malaysia in 2016 and screened for their antagonism against P. palmivora in vitro. The best two isolates AS1 and AS2 with more than $80 \%$ inhibition of radial growth (PIRG) were selected for subsequent experiments. Sequence analysis of the $16 \mathrm{~S}$ rRNA region indicated that these two isolates belonged to Pseudomonas aeruginosa (AS1) and Chryseobacterium proteolyticum (AS2). Bioactive volatile compounds were identified using gas chromatography-mass spectrometry (GCMS). Major compounds present in P. aeruginosa extract were identified as Eicosane $(9.11 \%)$, Hexatriacontane $(6.87 \%)$, Tetratetracontane $(5.17 \%)$, trans-2-Decenoic acid (17.04\%) and 1Phenanthrenecarboxylic acid, 1,2,3,4,4a,9,10,10a-octahydro-1,4a-dimethyl-7-(1-methylethyl) (3.60\%). In C. proteolyticum extract, major compounds were identified as Eicosane (11.29\%), Tetratetracontane (10.82\%), Heneicosane (10.78\%), Hexatriacontane (9.04\%) and Phenol, 2,4bis(1,1-dimethylethyl) (5.92\%). Effectiveness of P. aeruginosa and C. proteolyticum in reducing black pod lesion was confirmed on detached cocoa pods with $100 \%$ inhibition for both isolates. These results indicated that these two bacterial isolates have potential to be used as bio-control agents against P. palmivora.
\end{abstract}

Keyword: Biological control; Endophytic bacteria; GCMS; 16S rRNA region 